\title{
OPEN Conformational plasticity underlies membrane fusion induced by an HIV sequence juxtaposed to the lipid envelope
}

\begin{abstract}
Igor de la Arada ${ }^{1,5}$, Johana Torralba1,2,5, Igor Tascón ${ }^{1,3}$, Adai Colom ${ }^{1,2,3}$, Iban Ubarretxena-Belandiaa, ${ }^{1,3}$, José L. R. Arrondo ${ }^{1,2}$, Beatriz Apellániz ${ }^{4}$ \& José L. Nieva ${ }^{1,2}$

Envelope glycoproteins from genetically-divergent virus families comprise fusion peptides (FPs) that have been posited to insert and perturb the membranes of target cells upon activation of the virus-cell fusion reaction. Conserved sequences rich in aromatic residues juxtaposed to the external leaflet of the virion-wrapping membranes are also frequently found in viral fusion glycoproteins. These membrane-proximal external regions (MPERs) have been implicated in the promotion of the viral membrane restructuring event required for fusion to proceed, hence, proposed to comprise supplementary FPs. However, it remains unknown whether the structure-function relationships governing canonical FPs also operate in the mirroring MPER sequences. Here, we combine infrared spectroscopy-based approaches with cryo-electron microscopy to analyze the alternating conformations adopted, and perturbations generated in membranes by CpreTM, a peptide derived from the MPER of the HIV-1 Env glycoprotein. Altogether, our structural and morphological data support a cholesterol-dependent conformational plasticity for this HIV-1 sequence, which could assist cell-virus fusion by destabilizing the viral membrane at the initial stages of the process.
\end{abstract}

During the early phase of the replication cycle, the Human Immunodeficiency Virus type-1 (HIV-1) particle fuses its lipid envelope with the plasma membrane of the $\mathrm{CD} 4^{+}$target cell ${ }^{1,2}$. The reaction is triggered after engagement of the envelope glycoprotein (Env) with the cell receptor (CD4)/co-receptor (CXCR4 or CCR5), a specific recognition process that activates further refolding of the metastable native Env. Fusion activity of Env depends on the presence of the fusion peptide (FP), a conserved sequence located at the N-terminus of the transmembrane subunit gp41 (reviewed in Ref. ${ }^{3,4}$ ). Following fusion triggering, the FP is propelled towards the target cell membrane and embeds therein due to its hydrophobic character ${ }^{1,2}$. Subsequently, helical regions within gp41 ectodomains refold into an energetically stable, trimeric 6-helix bundle (6-HB), whose formation brings together the merging membranes: the plasma membrane of the target cell, and the lipid envelope of the virus (Fig. 1, see also Supplementary Fig. 1) ${ }^{5-7}$.

In analogy to FPs, insertion into the viral membrane of a C-terminal gp41 sequence known as the MembraneProximal External Region (MPER), is postulated to further contribute to the overall process of membrane merger (Fig. 1, see also Supplementary Fig. 1) ${ }^{8-13}$. This sequence, sitting at the bottom of the Env complex is exceptionally enriched in aromatic residues that promote interactions with the membrane interface (reviewed in Ref. $\left.{ }^{14-17}\right)$. MPER insertion may be disruptive for the viral membrane, since peptides and constructs derived from its sequence have been shown to exert virucidal effects ${ }^{18-21}$. The discovery that a number of antibodies targeting MPER can block membrane activity and infection, further underlines the importance of this region for the fusogenic function of the Env glycoprotein (reviewed in Ref. ${ }^{16,22}$ ).

There is structural evidence to support that the carboxy-terminal MPER sequence can combine with transmembrane domain (TMD) residues of gp41 to form a continuous helix, at least in one of the conformational states that are accessible to the pre-fusion Env complex ${ }^{23-30}$. A peptide that spans this helix (CpreTM, Fig. 1b), has

\footnotetext{
${ }^{1}$ Instituto Biofisika (CSIC-UPV/EHU), University of the Basque Country (UPV/EHU), PO Box 644, 48080 Bilbao, Spain. ${ }^{2}$ Department of Biochemistry and Molecular Biology, University of the Basque Country (UPV/EHU), PO Box 644, 48080 Bilbao, Spain. ${ }^{3}$ Ikerbasque, Basque Foundation for Science, 48013 Bilbao, Spain. ${ }^{4}$ Department of Physiology, Faculty of Pharmacy, University of the Basque Country (UPV/EHU), Paseo de la Universidad, 7, 01006 Vitoria-Gasteiz, Spain. ${ }^{5}$ These authors contributed equally: Igor de la Arada and Johana Torralba. ${ }^{\square}$ email: joseluis.nieva@ehu.es
} 


\section{a}

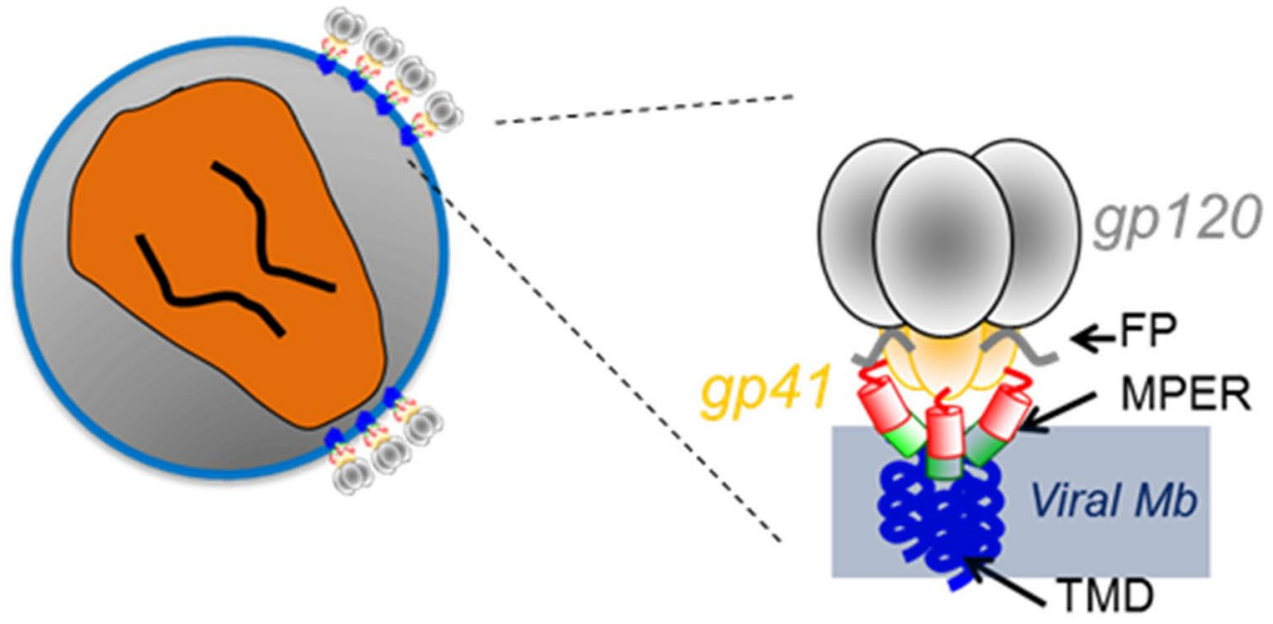

Env

b

FP NHR Loop CHR MPER TMD
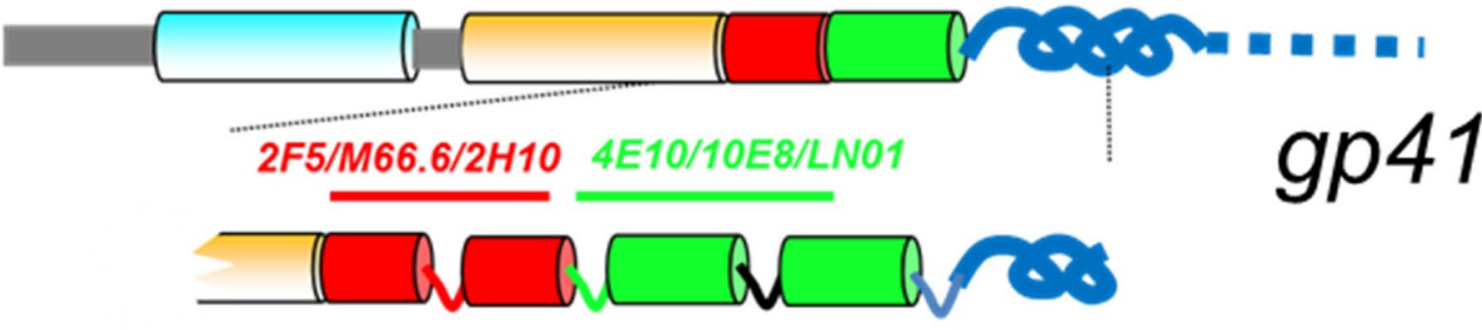

D664 N671 K683 G690

CpreTM

\section{KKK-NWFDITNWLWYIKLFIMIVGGLV-KK}

Figure 1. Designation of the HIV-1 CpreTM sequence used in this study. (a) Schematic displaying the general organization of the HIV-1 Env glycoprotein in virions (pre-fusion state). (b) Diagram showing the constituents of the gp41 subunit ectodomain and transmembrane anchor. Functional domains designated within its sequence include FP, fusion peptide; NHR and CHR, amino- and carboxy-terminal helical regions, respectively; MPER, membrane-proximal external region; TMD, transmembrane domain (see also Supplementary Fig. S1). The MPER-TMD region contains epitopes for the recognition of several broadly neutralizing HIV antibodies as indicated. The CpreTM sequence that derives from this region is shown below.

been shown to induce lipid bilayer restructuring upon partitioning into cholesterol (Chol)-enriched virus-like membranes ${ }^{21,31-33}$. However, it remains to be established whether structure-function relationships displayed by the standard N-terminal FP of gp41, also apply to this MPER-derived C-terminal sequence.

Cumulative experimental work using synthetic surrogates and model membranes has delineated physiologically relevant aspects of the FP function (reviewed in Ref. ${ }^{434,35}$ ). High-resolution NMR studies reveal the adoption of continuous $\alpha$-helical structures in membrane mimics ${ }^{36,37}$, whereas the combination of Infrared (IR) and Solid-State Nuclear Magnetic Resonance (SS-NMR) spectroscopy demonstrates that the $\alpha$-helical conformation can convert into oligomeric $\beta$ strand structures, a transition promoted by peptide density and the increase of the Chol concentration in the membrane ${ }^{38-40}$. Attenuated Total Reflectance (ATR)-IR studies further indicate that monomeric FP $\alpha$-helices penetrate into lipid bilayers in an oblique angle ${ }^{41,42}$, while its oligomeric $\beta$-strand counterparts appear to associate with the main axis forming a $90^{\circ}$ angle with respect to the membrane normal ${ }^{43}$. 
Thus, regarding its conformational behavior, a hallmark of membrane-bound HIV FP appears to be its plasticity, which enables the transition from inserted $\alpha$-helices, tilted relative to the bilayer normal, into extended $\beta$-strands, lying almost parallel to the membrane plane $e^{3,439-41,43-47}$. Studies in model systems suggest that these alternating conformations of the FP sequence can disrupt the lipid bilayer, breaching its permeability barrier and/or inducing aggregation and inter-bilayer mixing of lipids ${ }^{40,44,47-50}$. Moreover, the membrane-inserted FP appears to modulate the elastic properties of the bilayer and facilitate the formation of the non-bilayer lipid intermediates required for fusion ${ }^{51-54}$. In this context, the HR1 and HR2 helical domains within the ectodomain of gp41 are conceived as a mechanical device that brings the host-cell plasma membrane, primed for merger by the inserted FP, into contact with the viral membrane (Supporting Fig. S1).

In this work, we combine conventional IR spectroscopy, two-dimensional correlation IR spectroscopy (2D-COS-IR), and ATR-IR, to analyze the conformation and orientation adopted by the CpreTM peptide upon reconstitution into lipid bilayers. In line with the notion that the sequences flanking the TMD anchors of fusion glycoproteins are endowed with a degree of conformational plasticity ${ }^{55-57}$, our data reveal that the CpreTM helix can adopt membrane-inserted $\alpha$-helical structures that convert primarily into an extended $\beta$-strand conformation in Chol-rich membranes. Occurrence of the extended conformation lying parallel to the membrane plane correlates with the induction of vesicle fusion as visualized by cryo-electron microscopy (cryo-EM) of vitrified specimens. Thus, we conclude that CpreTM bound to membranes displays structural features of canonical FPs, and propose a structure-based mechanistic model that couples CpreTM helix unfolding to membrane merger during the HIV-1 fusion cascade.

\section{Results}

CpreTM conformation in a low-polarity medium. Before establishing the membrane-bound conformations of the CpreTM peptide, we analyzed the secondary structure adopted in a medium that mimics the low-polarity of lipid bilayers. As a reference we employed the published NMR structure of monomeric CpreTM in buffer containing 25\% (v/v) 1,1,1,3,3,3-hexafluoro-2-propanol (HFIP) ${ }^{24}$. Figure 2a displays the superposition of the calculated models for the CpreTM structure in HFIP, and a representative single model (left and right panels, respectively). All calculated models were consistent with a predominantly a-helical geometry, with evidence for disordered regions limited to the $\mathrm{COOH}$ and $\mathrm{NH}_{2}$ extremities. Panel b displays the circular dichroism (CD) spectra obtained for CpreTM in buffers with increasing HFIP content, and the quantitative analysis of the secondary structure composition ${ }^{58}$ (left and right panels, respectively). Consistent with peptide aggregation in solution, $\beta$-strands and turn/coil structures dominated the spectra at the lowest HFIP concentration $(2.5 \% \mathrm{v} / \mathrm{v})$, and diminished upon decreasing polarity. At the highest HFIP concentration where monomers are expected to be favored $(25 \% \mathrm{v} / \mathrm{v})$, the $\alpha$-helix contribution was predominant (ca. $65 \%)$, whereas only a residual signal from peptide aggregation remained. In these samples, components attributable to the disordered conformations and turns amounted to ca. $30 \%$.

Matching those findings, band decomposition of IR spectra obtained in 25\% HFIP identified a majority of amide-I vibrational modes arising from helical conformers, with bands centered at $1665 \mathrm{~cm}^{-1}\left(3_{10}\right.$-helix), $1655 \mathrm{~cm}^{-1}$ ( $\alpha$-helix) and $1630 \mathrm{~cm}^{-1}$ ( $\alpha$-helix solvated) $)^{59-61}$ amounting to ca. $56 \%$ of the total band area (Fig. $2 \mathrm{c}$ ). Besides, a band centered at $1642 \mathrm{~cm}^{-1}$ (ca. 34\%) was ascribed to disordered coil structures, whereas that at $1677 \mathrm{~cm}^{-1}$ was attributed to turns (ca. 9\%). Again, consistent with the monomeric state of the peptide (Panel a), the contribution of extended-aggregated conformations was negligible in these samples $(<1 \%)$.

CpreTM conformation in lipid bilayers. We next reconstituted CpreTM in membranes by co-mixing it with lipids in organic solvent, followed by gentle evaporation and hydration (see Materials and Methods). Figure 3 compares the IR spectrum of CpreTM in solution with that obtained after reconstitution in lipid bilayers made of 1-palmitoyl-2-oleoyl-sn-glycero-3-phophocholine (POPC) (top and bottom panels, respectively). The amide-I region of the IR spectrum in solution displayed a prominent band centered at $1622 \mathrm{~cm}^{-1}$, which together with high-frequency absorption in the $1680-1690 \mathrm{~cm}^{-1}$ region, denoted that a majority of peptide chains were unfolded/aggregated. In contrast, upon reconstitution in POPC bilayers, the maximum shifted to $1654 \mathrm{~cm}^{-1}$, whereas the contribution of the $1620 \mathrm{~cm}^{-1}$ band was irrelevant. In these samples predominant helical conformers amounted to ca. $70 \%$. Besides, in comparison with the absorption band components measured in HFIP (Fig. 2c), the contribution of turns and disordered chains decreased, whereas the amide-I band became overall narrower. These spectral variations reflect a reduction in the conformational space accessible to the CpreTM chain upon reconstitution in lipid bilayers, consistent with the majority of the membrane-associated peptide adopting a canonical $\alpha$-helical conformation.

Conformational changes in cholesterol-containing membranes. Cholesterol (Chol) is a major lipid of the HIV membrane required for virion infectivity ${ }^{62-66}$. Therefore, we analyzed the conformation adopted by CpreTM reconstituted in membranes containing increasing Chol concentrations. Figure 4a displays the series of raw and deconvolved IR spectra as a function of Chol content in membranes (left and right panels, respectively). A shoulder centered at ca. $1620 \mathrm{~cm}^{-1}$ could be already discerned in the samples that contained low Chol, which evidenced an initial accumulation of extended chains. Samples containing the highest Chol concentrations displayed a more conspicuous band centered at $1622 \mathrm{~cm}^{-1}$, consistent with a $\beta$-strand-like conformation dominating the secondary structure of the membrane-bound peptide under these conditions.

To get more insight into the CpreTM conformational changes induced by the membrane Chol concentration, we next performed the $2 \mathrm{D}$-correlation analysis of the IR spectra in the corresponding amide I band region ${ }^{67-69}$ (Fig. 4b). We note that relevant effects detected on the 2D maps often reflect subtle changes in the relative contents of the amide I band components. Therefore, in addition to the $\beta$-strand band that dominates in samples 
a
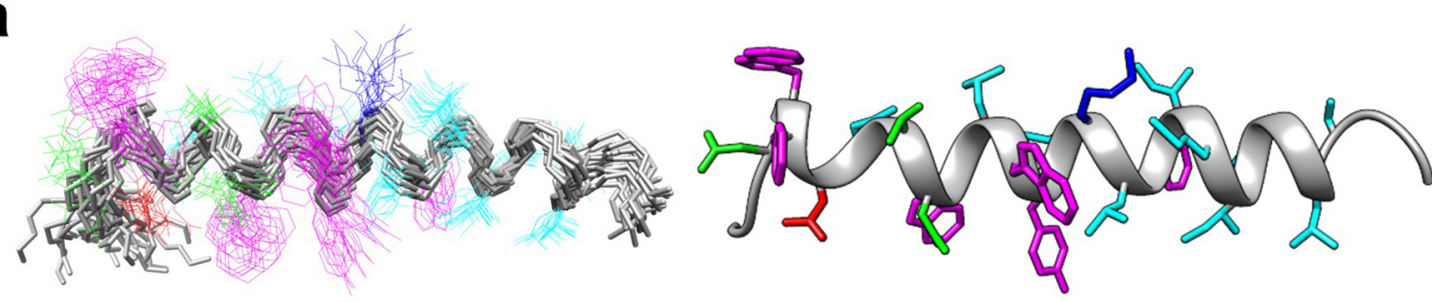

b
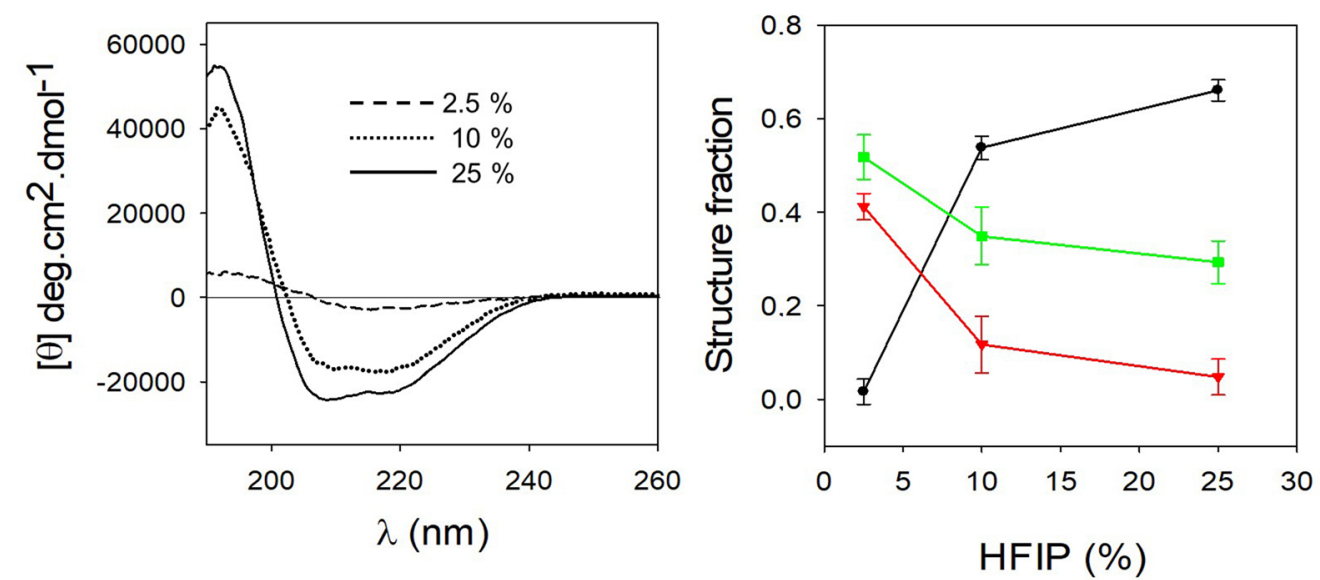

C

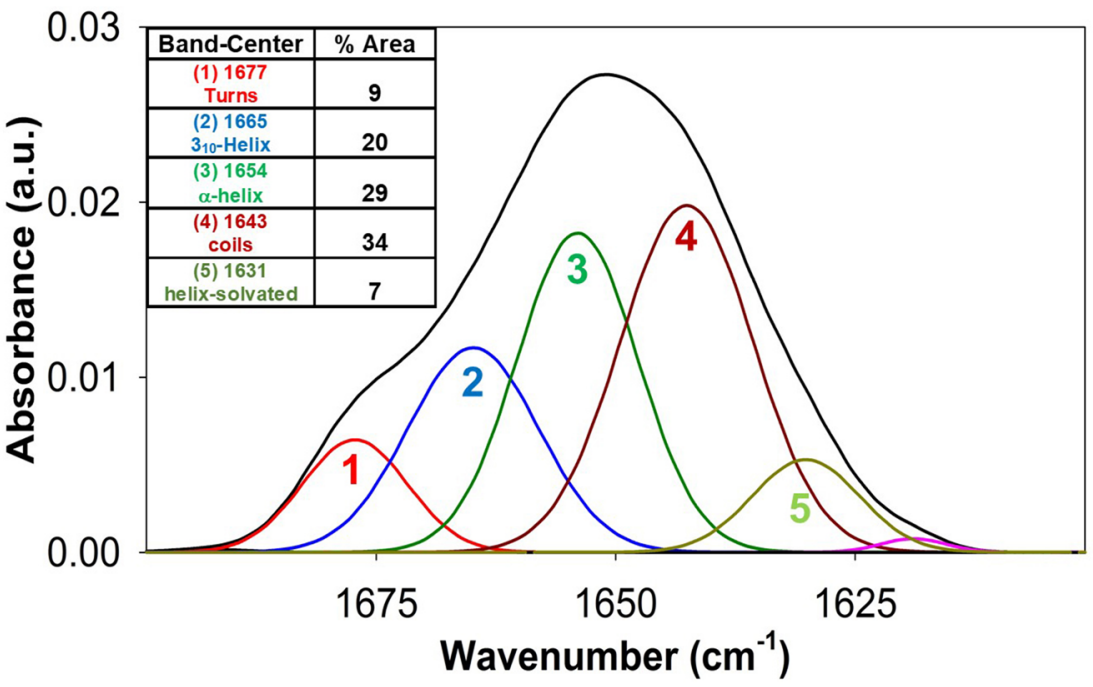

Figure 2. Structural analysis in a low-polarity medium. (a) NMR structure of the CpreTM peptide solved in 25\% 1,1,1,3,3,3-hexafluoro-2-propanol (HFIP) (v/v) (PDB ID: 2MG2). (b) Left: CD spectra obtained at $25^{\circ} \mathrm{C}$ in media containing increasing amounts of HFIP as indicated in the panel; Right: secondary structure fractions estimated from $\mathrm{CDPro}^{83}$. Means \pm the standard deviation for the fraction values estimated with CONTIN-LL, CDSSTR, and SELCON3 are plotted. Black dots, red triangles and green squares depict the fractions of helix, strand and the sum of turns + unordered conformations, respectively. (c) IR spectrum in the amide I region obtained in buffer containing 25\% HFIP (v/v). The absorption band was decomposed into different components. The original spectrum and the sum of the band components are superimposed and indistinguishable. The inset displays the secondary structure assignation for the main components (bands labeled with numbers 1 to 5) and the area percentages (rounded off to the nearest integer).

containing high concentrations of Chol (centered at ca. $1620 \mathrm{~cm}^{-1}$ ), the analysis also revealed the evolution of the rest of the spectral components i.e., bands centered at ca. 1675, 1660, 1650, 1642 and $1635 \mathrm{~cm}^{-1}$.

In the synchronous $(\Phi) 2 \mathrm{D}$ maps of CpreTM (Fig. 4b, top panels), autopeaks indicated simultaneous changes in the bands composing the amide-I spectrum. In the $2 \mathrm{D}$ maps of the raw spectra (left), autopeaks were found centered at 1650 and $1620 \mathrm{~cm}^{-1}$, whereas the single cross-relation negative peak $1620 / 1650 \mathrm{~cm}^{-1}$ reflected that both vibrations were affected in-phase by Chol, the first component augmenting in intensity, the second diminishing. Higher resolution was attained using the $\Phi$ map based on the deconvolved spectra (right). Particularly, all 


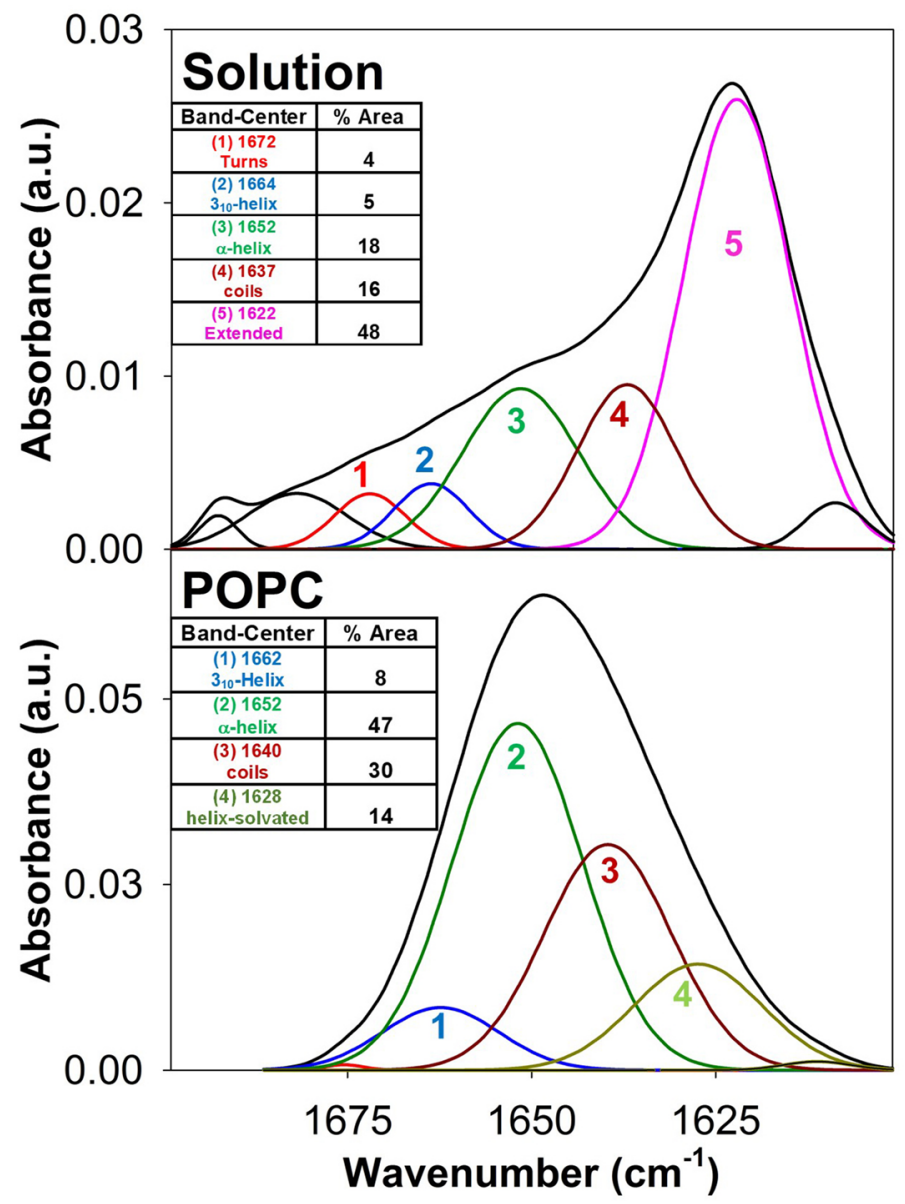

Figure 3. Reconstitution of CpreTM in lipid bilayers. Top and bottom IR spectra display respectively the amide I components measured in buffer or after reconstitution of the peptide in POPC lipid bilayers (Peptide-to-lipid ratio, 1:50). Insets in both panels display the secondary structure assignation for the main band components and their area percentages.

helical components were evidenced as autopeaks centered at ca. $1635 \mathrm{~cm}^{-1}, 1655 \mathrm{~cm}^{-1}$ and $1665 \mathrm{~cm}^{-1}$, which could be observed together with cross-relation negative peaks $1620 / 1635 \mathrm{~cm}^{-1}, 1620 / 1655 \mathrm{~cm}^{-1}$ and $1620 / 1665 \mathrm{~cm}^{-1}$.

The corresponding asynchronous $(\Psi)$ maps reflected the sequential order of events induced by the increase of Chol (Fig. 4b, bottom panels) ${ }^{67-69}$. The asynchronous peaks were positive (red contours) if the change in the first frequency occurred accelerated with respect to that in the second one, and negative (blue contours) if delayed. The positive correlation peak $1655 / 1665 \mathrm{~cm}^{-1}$ detected in the raw-spectra maps, suggests the formation of less stable short regions deviating from canonical $\alpha$-helicity and adopting $3_{10}$-helical geometries, whereas the negative one at $1620 / 1665 \mathrm{~cm}^{-1}$ supports the conversion of the $3_{10}$-helix intermediates into extended strands.

This pathway was also apparent in the $\Psi$ map based on deconvolved spectra. In this case, an additional positive correlation peak was found for the pair $1635 / 1665 \mathrm{~cm}^{-1}$. It is known that partial solvation of $\alpha$-helical structures can give rise to low-frequency bands centered at ca. $1635-1630 \mathrm{~cm}^{-1}$ because of the cross-hydrogen bonds that can be formed with water ${ }^{60,61}$ (see also Fig. 2c). Thus, we attribute the CpreTM absorption mode at $1635 \mathrm{~cm}^{-1}$ to a fraction of the helical structure not buried in the membrane, i.e., exposed to solvent and/or in contact with interfacial polar moieties. The positive correlation found at $1635 / 1665 \mathrm{~cm}^{-1}$ suggests that these solvated helices also unfold adopting $3_{10}$-helical geometries, whereas the negative one at $1635 / 1655 \mathrm{~cm}^{-1}$ would be consistent with the buried helical fraction unfolding more readily than the solvent-exposed one upon increasing the Chol concentration.

Additional positive peaks were observed at $1655 / 1675 \mathrm{~cm}^{-1}$ and $1635 / 1675 \mathrm{~cm}^{-1}$, and a negative peak found at $1620 / 1675 \mathrm{~cm}^{-1}$. This indicates that $\beta$-turns can also act as intermediates of the helix-to-strand unfolding process. In conclusion, upon increasing the Chol content in the membrane, $\beta$-turns $/ 3_{10}$-helical regions seem to be produced at the expense of the canonical $\alpha$-helical conformations, and these intermediates appear to convert into extended strands.

To establish whether the C-terminal region accounted for the tendency of MPER to adopt extended conformations in membranes, we also analyzed the effect of Chol on the conformation adopted by NpreTM, a peptide overlapping with the aromatic-rich $\mathrm{N}$-terminal stretch of CpreTM, but lacking its TMD residues (Supplementary Fig. S2a). Following CpreTM's trend, NpreTM reconstituted in POPC membranes adopted a main 

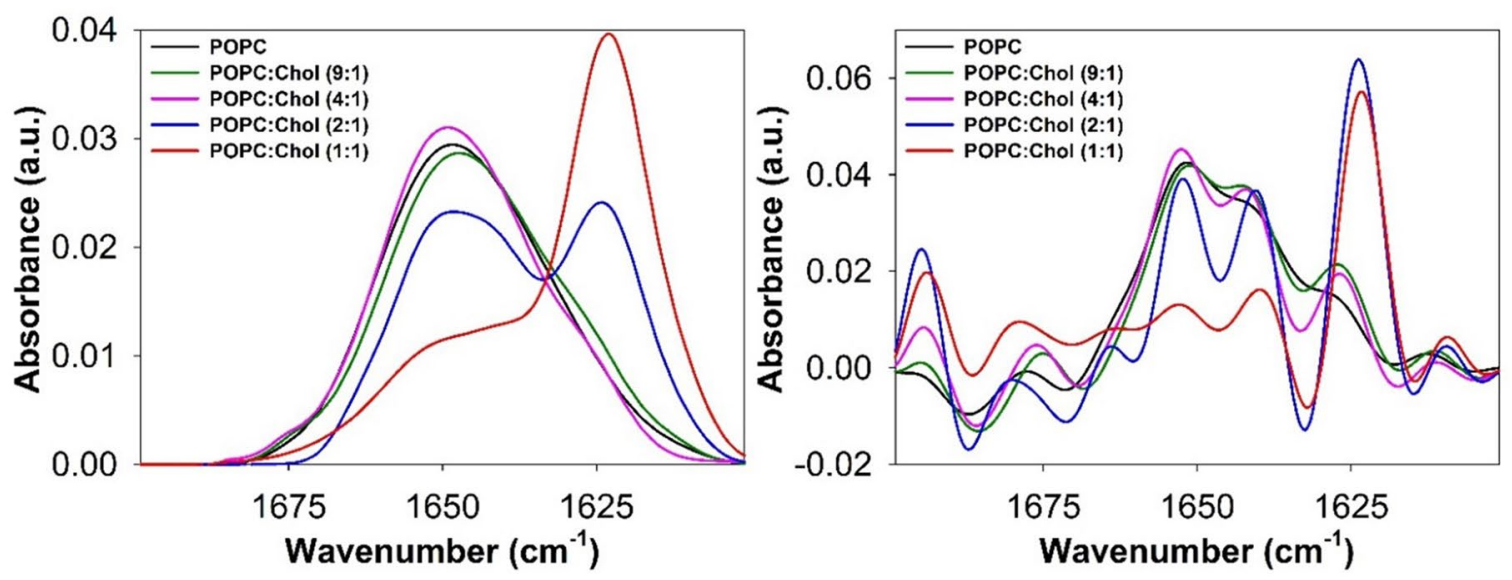

b

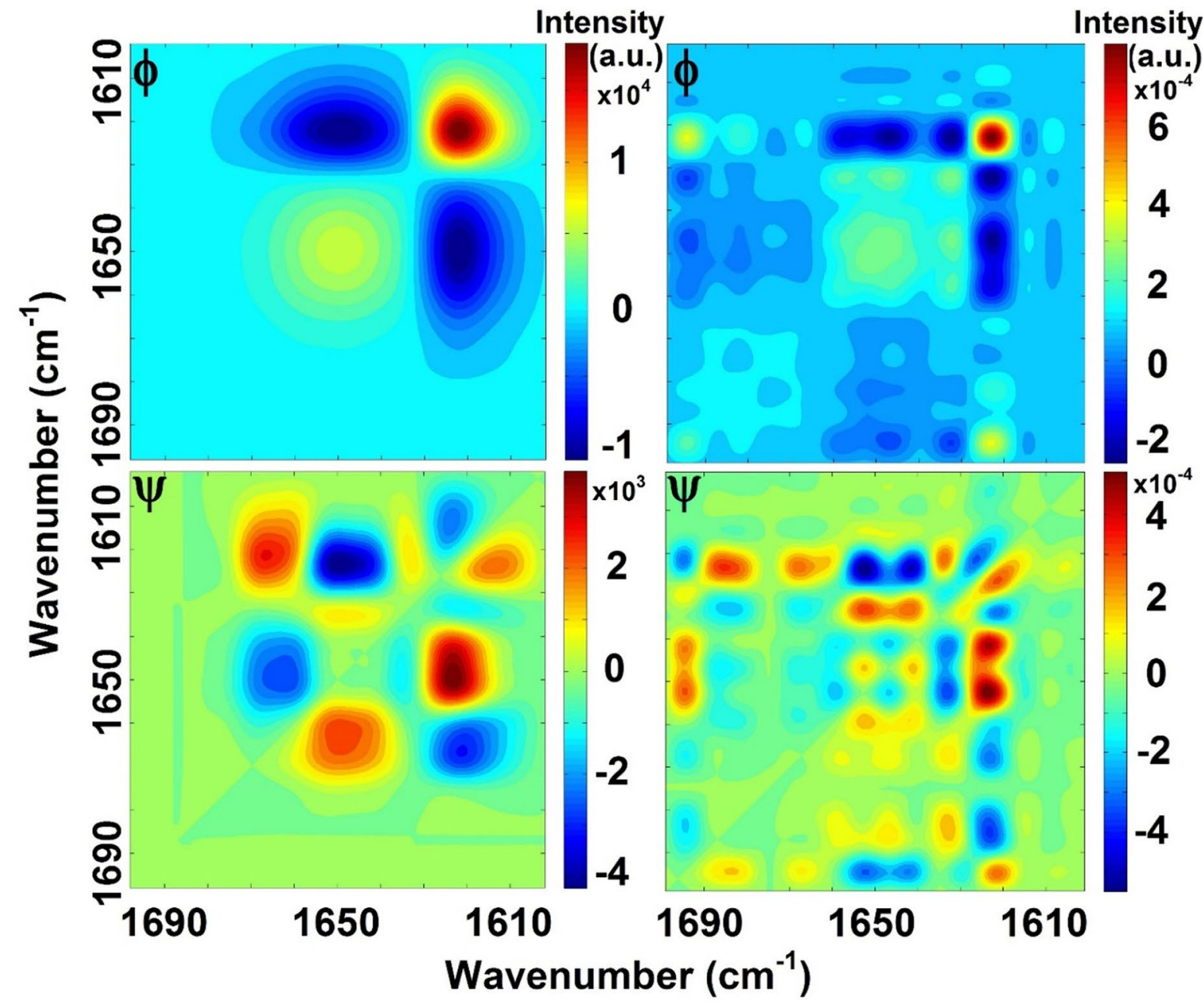

Figure 4. Conformations adopted by CpreTM as a function of the Chol content in membranes. (a) Amide I region IR spectra of CpreTM reconstituted in membranes (peptide-to-lipid ratio, 1:50) that contained increasing Chol concentrations as indicated in the panels. Raw and deconvolved spectra are shown (left and right panels, respectively). (b) 2D-COS IR analysis. Synchronous (top) and asynchronous (bottom) correlation map contours of the raw (left) and deconvolved (right) IR spectra obtained with increasing Chol concentrations are shown. Red peaks correspond to positive correlations and blue peaks to negative ones. 
a
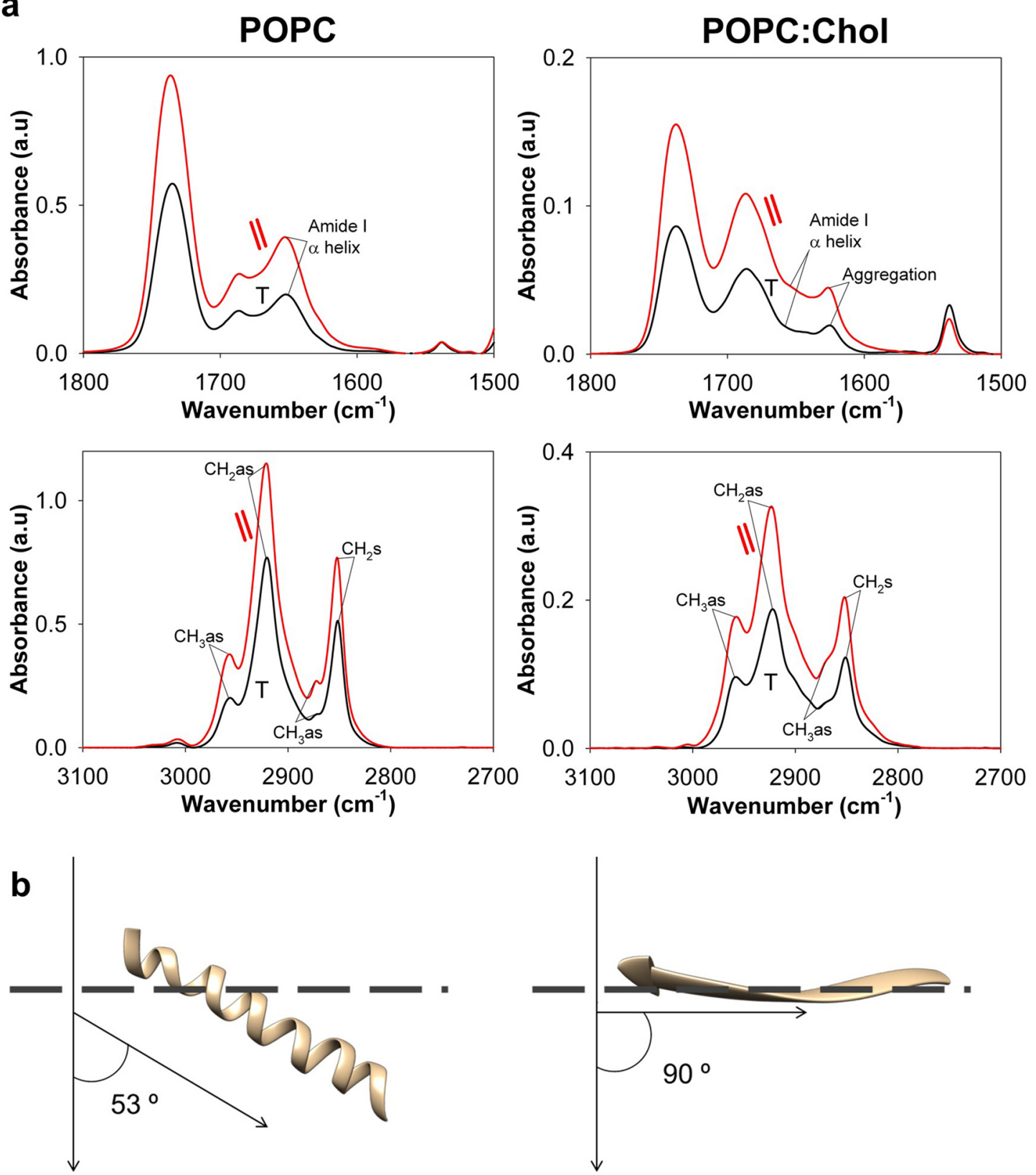

Figure 5. Angle of insertion of CpreTM main conformations as determined by ATR-IR. (a) Top: Comparison of ATR-IR spectra in the amide I region of CpreTM reconstituted in POPC or POPC:Chol (1:1) membranes (left and right panels, respectively) (peptide-to-lipid mole ratio, 1:50). Bottom: a similar comparison was made in the $\mathrm{CH}_{2} / \mathrm{CH}_{3}$ stretching region of the spectra. The main orientations of peptide and acyl chains are inferred from the ratio of peak areas recorded with incident light polarized parallel $(\|)$ and perpendicular $(\top)$ to the membrane normal (calculated values for the order parameters and tilt angles are displayed in Table 1). (b) Models for the membrane-associated structures and orientations adopted by CpreTM in POPC and POPC:Chol (1:1) membranes (left and right panels, respectively).

helical conformation (Supplementary Fig. S2b). However, when reconstituted in POPC:Chol (1:1) membranes, $\beta$-strands did not dominate the overall conformation of the NpreTM peptide, supporting the implication of the membrane-buried CpreTM TMD residues in the conformational conversion promoted by Chol.

Membrane insertion angle of alternating conformations. The previous results support the efficient lipid bilayer reconstitution of the CpreTM peptide as an $\alpha$-helix, and the possibility of its transitioning to extended structures in Chol-enriched membranes. Using ATR-IR spectroscopy, we next determined the tilt of these alternating CpreTM conformations relative to the membrane normal. ATR-IR absorbance spectra were measured using perpendicular and parallel polarized light (Fig. 5a). From these spectra, the experimental average dichroic ratios were determined, and order parameters $S$ and tilt angles calculated (Table 1$)^{70-72}$. 


\begin{tabular}{|c|c|c|c|c|c|c|}
\hline Wavenumber $\left(\mathrm{cm}^{-1}\right)$ & Vibration $^{\text {a }}$ & $\theta\left({ }^{\circ}\right)^{b}$ & Avg dichroic ratio \pm SD & $\operatorname{Avg} S \pm \mathrm{SD}^{\mathrm{c}}$ & $\operatorname{Avg} \gamma \perp \pm$ SD $^{d}$ & $\operatorname{Avg}^{\mathrm{L}} \pm \mathrm{SD}^{\mathrm{e}}$ \\
\hline \multicolumn{7}{|l|}{ Lipid alone (POPC) } \\
\hline 2920 & as $\mathrm{CH}_{2}$ stretching & 90 & $1.33 \pm 0.00$ & $0.48 \pm 0.00$ & $35.97 \pm 0.15$ & \\
\hline 2850 & $s \mathrm{CH}_{2}$ stretching & 90 & $1.19 \pm 0.00$ & $0.62 \pm 0.00$ & $32.28 \pm 0.20$ & \\
\hline 2870 & $s \mathrm{CH}_{3}$ stretching & 0 & $9.57 \pm 0.30$ & $0.69 \pm 0.01$ & $27.05 \pm 0.39$ & \\
\hline \multicolumn{7}{|l|}{ CpreTM+POPC } \\
\hline 2920 & as $\mathrm{CH}_{2}$ stretching & 90 & $1.50 \pm 0.01$ & $0.34 \pm 0.01$ & $41.60 \pm 0.15$ & \\
\hline 2850 & $s \mathrm{CH}_{2}$ stretching & 90 & $1.47 \pm 0.01$ & $0.36 \pm 0.01$ & $40.68 \pm 0.38$ & \\
\hline 2870 & $s \mathrm{CH}_{3}$ stretching & 0 & $5.88 \pm 0.33$ & $0.53 \pm 0.02$ & $34.07 \pm 0.86$ & \\
\hline 1656 & Amide I-helix & 30 & $2.03 \pm 0.03$ & $0.02 \pm 0.01$ & $54.13 \pm 0.55$ & $53.27 \pm 1.32$ \\
\hline \multicolumn{7}{|c|}{ Lipid alone (POPC:Chol) } \\
\hline 2920 & as $\mathrm{CH}_{2}$ stretching & 90 & $1.62 \pm 0.02$ & $0.24 \pm 0.01$ & $45.21 \pm 0.44$ & \\
\hline 2850 & $s \mathrm{CH}_{2}$ stretching & 90 & $1.50 \pm 0.00$ & $0.34 \pm 0.00$ & $41.53 \pm 0.04$ & \\
\hline 2870 & $s \mathrm{CH}_{3}$ stretching & 0 & $1.49 \pm 0.04$ & $-0.17 \pm 0.02$ & $61.95 \pm 0.75$ & \\
\hline \multicolumn{7}{|l|}{ CpreTM + POPC:Chol } \\
\hline 2920 & as $\mathrm{CH}_{2}$ stretching & 90 & $1.69 \pm 0.01$ & $0.20 \pm 0.01$ & $47.06 \pm 0.20$ & \\
\hline 2850 & $s \mathrm{CH}_{2}$ stretching & 90 & $1.62 \pm 0.01$ & $0.25 \pm 0.01$ & $44.99 \pm 0.38$ & \\
\hline 2870 & $s \mathrm{CH}_{3}$ stretching & 0 & $3.18 \pm 0.72$ & $0.24 \pm 0.12$ & $45.50 \pm 4.55$ & \\
\hline 1623 & Amide I-sheet & 70 & $2.42 \pm 0.15$ & $-0.33 \pm 0.11$ & $71.55 \pm 6.82$ & $90.00 \pm 0.00$ \\
\hline
\end{tabular}

Table 1. ATR-IR data of the CpreTM peptide. ${ }^{a}$ Vibrations are presented as symmetric $(s)$ or asymmetric (as). ${ }^{\mathrm{b}}, \theta$ direction of the dipole moment associated with the vibration with respect to the direction of the main molecular axis (aliphatic chain or peptide-secondary structure). ${ }^{c} S$, form factor. ${ }^{\mathrm{d}} \gamma \perp$, angle between the direction of the molecular axis and the perpendicular to the crystal plane (similar to the membrane plane). ${ }^{\mathrm{e}} \gamma^{\mathrm{L}}$, angle between the direction of the peptide-secondary structure axis and the calculated aliphatic chain axis.

According to the tilt angle inferred from the dichroic ratios, the longitudinal axis of the CpreTM helix formed an angle of $53^{\circ}$ with the POPC lipid bilayer normal (Fig. 5b). Angles of a comparable magnitude (ca. $50^{\circ}$ ) have been reported in the literature for the HIV-1 and SIV FPs inserted into lipid bilayers ${ }^{42,46}$. Thus, our ATR-IR data support that, similarly to the $\mathrm{N}$-terminal FP, the C-terminal Env sequence covered by CpreTM could insert in a tilted orientation into the membrane at some stage during the fusion process. Conversely, the CpreTM $\beta$-strands oriented almost parallel to the membrane plane in the POPC:Chol (1:1) sample (angle of ca. $90^{\circ}$ with respect to the normal) (Fig. 5b), also in accordance with data reported in the literature for the N-terminal FP under fusogenic conditions $s^{39,43,44}$.

Changes in vesicle morphology induced by CpreTM reconstituted in membranes. Despite the differences in the attained conformation, the reconstituted CpreTM peptide incorporated to the same extent and quantitatively into vesicles containing different concentrations of Chol (Fig. 6a). In contrast, Cryo-EM images revealed different morphologies for the peptide-containing vesicles, suggesting that the adopted conformations induced distinct patterns of membrane destabilization (Fig. 6b,c and Supplementary Fig. S3). Untreated control samples displayed spherical vesicles with heterogeneous sizes ranging in mean diameter from ca. 100 to $200 \mathrm{~nm}$ (Fig. 6b,c, bottom panels). The $a$-helical peptide did not alter the overall morphology or size of POPC vesicles when incorporated at a 1:50 peptide-to-lipid dose (Fig. 6b,c, top left panels). In contrast, an increase of extended conformations in the peptide-treated samples (Chol-containing membranes) correlated with a significant increase of the mean vesicle size (Fig. 6b,c, top center and right panels). Particularly, in peptide-containing POPC:Chol (1:1) samples, massive aggregation and vesicle sizes in the range of $500 \mathrm{~nm}$ could be observed.

The Supplementary Figure S3 displays more detailed views of the effects exerted by CpreTM on vesicle morphology. The peptide reconstituted in POPC membranes did not affect the stability of the vesicle samples, whereas its inclusion into POPC:Chol membranes induced tight vesicle-vesicle contacts (lipid bilayer aggregation) and increased the mean diameter of the vesicles (membrane fusion). Notably, POPC:Chol (1:1) vesicles containing the reconstituted NpreTM control peptide displayed a pattern of tight bilayer aggregation, which did not result in an increase of the mean vesicle size (Supplementary Fig. S2c). Thus, it appears that completion of the fusion process required the presence of the CpreTM TMD residues.

Lipid aggregates with spongy morphology also accumulated sporadically in certain areas of CpreTM-containing POPC:Chol vesicles (Supplementary Fig. S3, bottom panels). The occurrence of lipid aggregates reminiscent of non-lamellar arrangements suggested that, following an FP-like fashion ${ }^{4,51-54}$, the CpreTM peptide could also facilitate the formation of highly curved lipid structures involved in membrane merger ${ }^{35}$. However, control experiments using ${ }^{31} \mathrm{P}-\mathrm{NMR}$ failed to detect evidence for the promotion of this type of non-lamellar arrangements in peptide-containing POPC:Chol (1:1) membranes (Supplementary Fig. S4). Moreover, an inspection by Atomic Force Microscopy (AFM) of supported lipid bilayers containing reconstituted peptide revealed that CpreTM disrupted the lipid continuity of the solvent-accessible membrane monolayer, and increased the amount of force required to break the bilayer (Supplementary Fig. S5). Thus, it appears that inclusion of CpreTM at doses leading to vesicle fusion did not facilitate membrane deformation by increasing curvature or reducing bilayer stiffness $s^{35}$. 
a

POPC

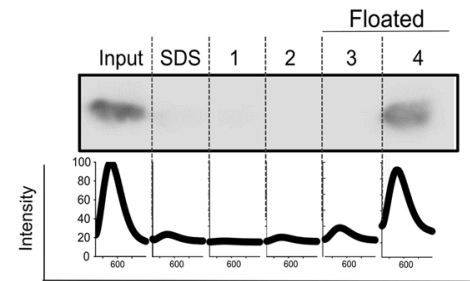

$\beta$-strand: $<1 \%$

b
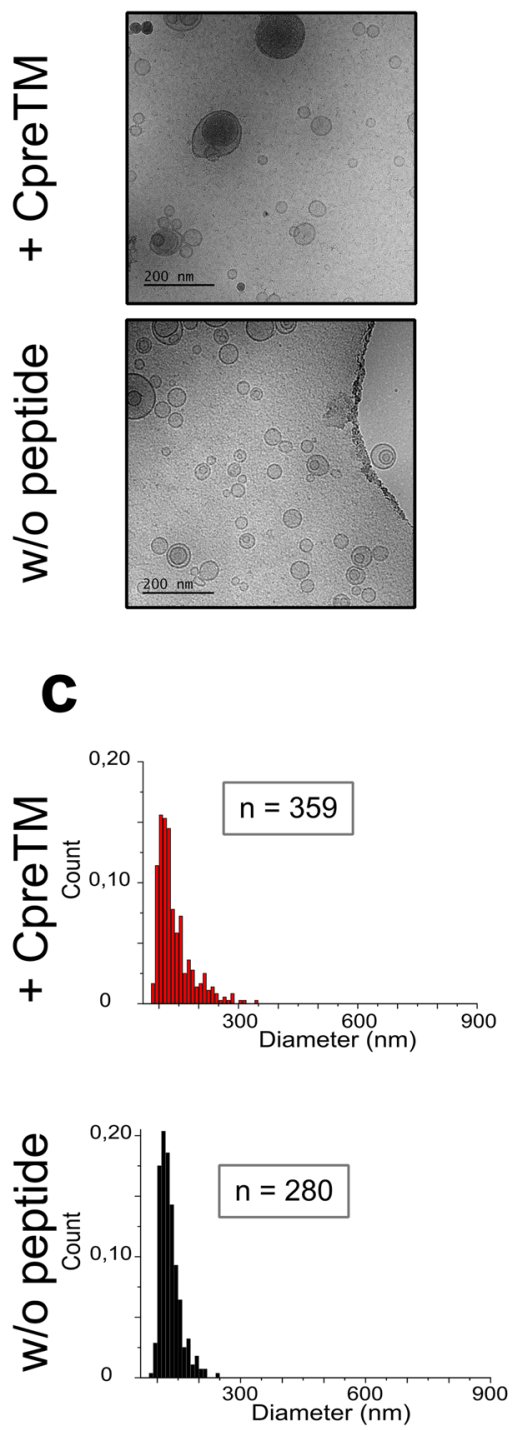

POPC:Chol (2:1)

POPC:Chol (1:1)

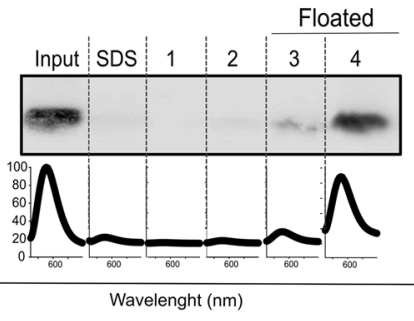

ß-strand: $39 \%$
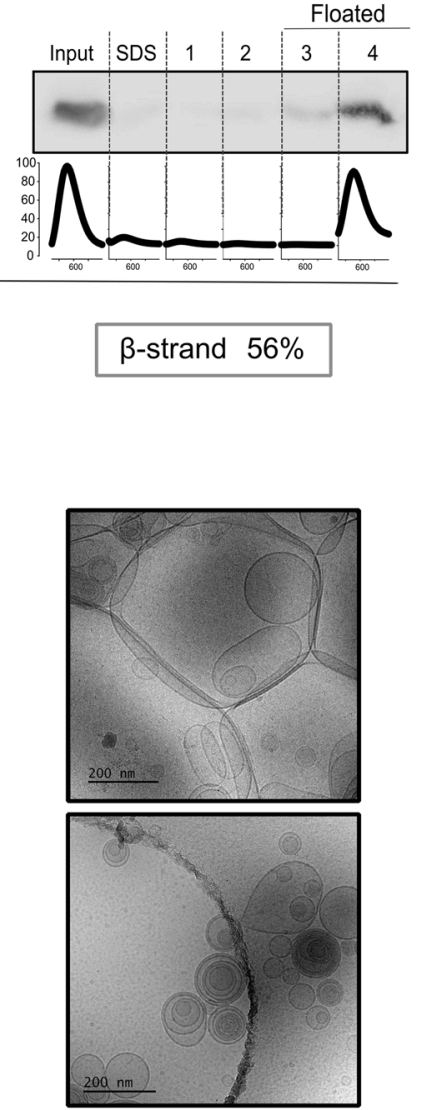
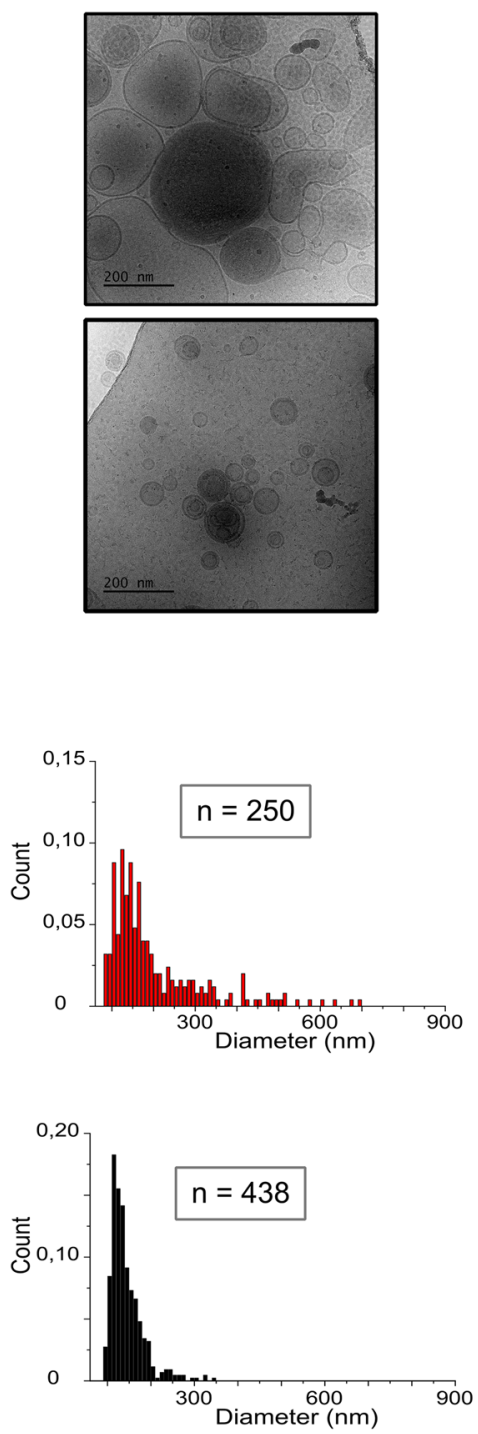
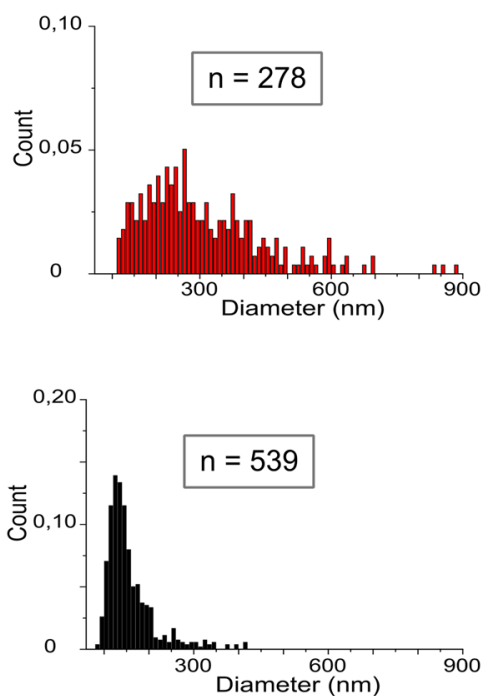

Figure 6. Vesicle morphology changes induced by the different conformations adopted by CpreTM in membranes. (a) Vesicle flotation analysis. Incorporation to vesicles of CpreTM was verified after ultracentrifugation in a sucrose gradient (peptide-to-lipid mole ratio, 1:50). The presence of the peptide in the floated and non-floated fractions of the gradient and in the original sample (input) was revealed by Western Blot analysis after Tris-Tricine SDS-PAGE separation. Fluorescence emission spectra below reveal the presence of Rhodamine-labeled vesicles in the collected fractions. The amount of extended $\beta$-strand adopted by the peptide in the samples is indicated below the panels (values determined in the spectra of Fig. 5a after band decomposition). (b) Vesicle morphology by cryo-EM. Images obtained for vesicles containing reconstituted peptide are shown in the top-panels. Bottom panels display images of vesicles devoid of peptide. (c) Vesicle size distribution as determined from the diameters measured in cryo-EM images. The sizes of oval-shaped vesicles were determined by measuring their major axes. Conditions as those in the previous panel. 
a

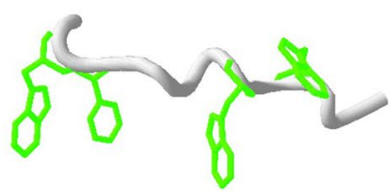

2PV6: NWFNITNWLWYIK

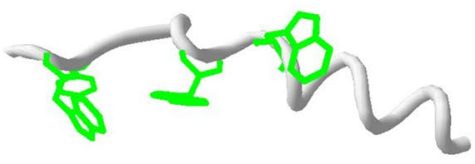

2MG3: NWFDITNWLWYIKLFIMIV

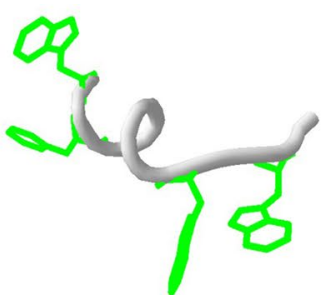

2X7R: NWFNITNWLWY
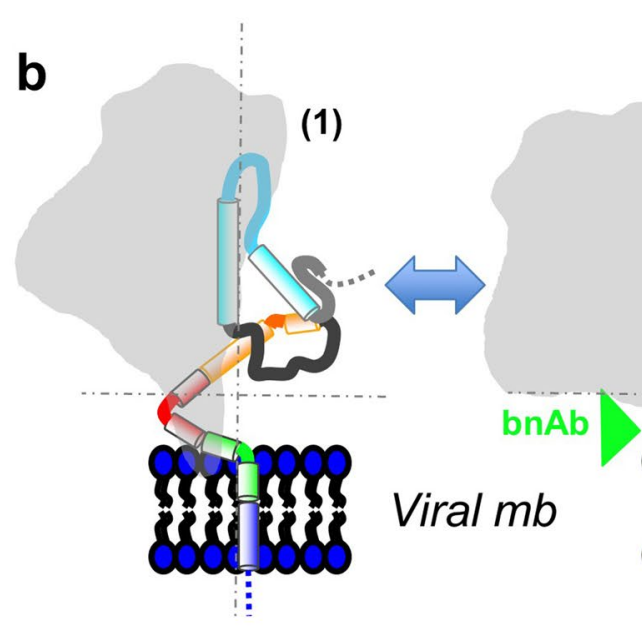
The model displayed in Fig. 7b integrates these findings in a general model of HIV-1 gp41-induced membrane fusion. The prefusion Env complex may alternate compact (1) with more open conformations (2), and it is likely that in these structures the helices spanning the MPER-TMD sequence could kink at different positions. Recent structural studies support that, at least in one of those conformational states, a straight and continuous CpreTM helix inserted in a subtle angle would be the target to antibodies exerting broad and potent neutralization ${ }^{29,30}$.

Subsequent activation of the fusion process involves the refolding of the gp41 helical domains HR1 and HR2 (depicted in cyan and yellow colors, respectively) to initiate the formation of a compact 6-HB. Establishment of the extensive helix-helix hydrophobic interactions between HR1 and HR2 implies the relocation of the helical sections and the reorientation of the complex main axis with respect to the membrane (3). We infer that the initial formation of the 6-HB likely requires extension of the Ca chain at sections joining the emerging complex to the membrane-inserted sequences. These extended hydrophobic chains, most prominently at FP and MPER areas, could associate with membrane surfaces helping to overcome repulsive hydration and electrostatic forces, as the cell and viral membranes approach pulled by the growing 6-HB hairpin. Furthermore, our data suggest that the CpreTM sequence could also break lipid continuity of the viral membrane external monolayer (Supplementary Fig. S5), generating poorly solvated hydrophobic spots where the initial contacts could be established between the approaching bilayers.

Overall, the experimental data presented in this study support the notion that a similar conformational plasticity underpins the membrane activity of the FP and CpreTM region during the initial (and transient) stages of HIV-1 fusion, but caution that effects of these sequences on the elastic properties of membranes involved in the process might be different. In this regard, we note that the present work provides no hints as to how the membrane-inserted structures of the FP or MPER evolve at later stages of the fusion process. It has been argued that the FP could first assemble as $\beta$-sheets on membrane surfaces, but later convert into $\alpha$-helices to complete fusion $^{38}$. Thus, at least theoretically, it is possible that at later stages of the fusion process the CpreTM sequence also attains secondary structures and membrane topologies that differ from those described in this work. Such alternative conformations might allow completion of the 6-HB structure and/or modulate the elastic properties of the membrane to facilitate fusion ${ }^{54}$.

\section{Materials and methods}

Reagents. The peptide sequence derived from the gp41 MPER-TMD region, KKK-NWFDITNWLWYIKLFIMIVGGLV-KK (CpreTM) (Fig. 1) was produced by solid-phase synthesis using Fmoc chemistry as C-terminal carboxamides and purified by HPLC (estimated purity 97\%). 1-palmitoyl-2-oleoyl-sn-glycero3-phophocholine (POPC) and cholesterol (Chol) were purchased from Avanti Polar Lipids (Birmingham, AL, USA). N-(lissamine Rhodamine B sulfonyl) phosphatidylethanolamine (N-Rh-PE) was from Thermo Fisher Scientific (Waltham, Massachusetts, USA). 1,1,1,3,3,3-hexafluoro-2-propanol (HFIP) was obtained from SigmaAldrich (St. Louis, Missouri, USA). Monoclonal antibody 4E10 (MAb4E10), kindly donated by D. Katinger (Polynum Inc., Vienna, Austria), and rabbit anti-human-IgG-HRP (Santa Cruz Biotechnologies) were used to reveal the membrane-bound peptide.

CpreTM reconstitution in membranes. To prepare CpreTM-containing vesicles, adequate amounts of lipids and peptide were mixed in organic solvent prior to the production of the liposomes as described ${ }^{82}$. Briefly, phospholipid and cholesterol were dissolved in chloroform:methanol 1:2 (vol:vol) and mixed with CpreTM (dissolved in $100 \%$ ethanol) at a peptide-to-lipid molar ratio of 1:50. The mixture was dried under a $\mathrm{N}_{2}$ stream followed by $2 \mathrm{~h}$ vacuum pumping to remove traces of organic solvents. Subsequently, the dried lipid films were subjected to $2 \mathrm{~h}$ of gentle hydration with $\mathrm{H}_{2} \mathrm{O}$ using a $\mathrm{N}_{2}$ gas bubbler to facilitate dispersion of the dried lipidpeptide film in PBS buffer. Next, the multilamellar vesicles were bath sonicated $\left(1 \mathrm{~h}, 55^{\circ} \mathrm{C}\right)$ and subjected to 15 freeze and thaw cycles to obtain unilamellar vesicles. Finally, effective incorporation of the peptide to the vesicles was ensured by peptide flotation coupled to that of lipid vesicles after ultracentrifugation of the samples in a sucrose gradient as described ${ }^{32}$.

Circular dichroism. Circular dichroism (CD) measurements were carried out on a thermally-controlled Jasco J-810 circular dichroism spectropolarimeter calibrated routinely with (1S)-(+)-10-camphorsulfonic acid, ammonium salt. CpreTM stock samples dissolved in DMSO, were lyophilized and subsequently dissolved in an aqueous buffer ( $2 \mathrm{mM}$ Hepes, $\mathrm{pH}, 7.4$ ) at $0.03 \mathrm{mM}$ concentration with $2.5 \%, 10 \%$ or $25 \%$ (v:v) 1,1,1,3,3,3-hexafluoro-2-propanol (HFIP). Spectra were measured in a $1 \mathrm{~mm}$ path-length quartz cell equilibrated at $25^{\circ} \mathrm{C}$. Data were taken with a $1 \mathrm{~nm}$ band-width, $100 \mathrm{~nm} / \mathrm{min}$ speed, and the results of 20 scans per sample were averaged. Quantitative analysis of the spectra was carried out using the CDPro software ${ }^{83}$, as previously described ${ }^{58}$.

Transmission infrared spectroscopy. Infrared spectra were recorded in a Thermo Nicolet Nexus 5700 (Thermo Fisher Scientific; Waltham, MA) spectrometer equipped with a mercury-cadmium-telluride detector using a Peltier based temperature controller (TempCon, BioTools Inc., Wauconda, IL) with calcium fluoride cells (BioCell, BioTools Inc., Wauconda, IL). CpreTM-containing samples were lyophilized and subsequently prepared at $3 \mathrm{mg}$ (peptide)/mL in $\mathrm{D}_{2} \mathrm{O}$ buffer (PBS). A $25 \mu \mathrm{l}$ sample aliquot was deposited on a cell that was sealed with a second cell. Reference windows without peptide were prepared similarly. Typically 370 scans were collected for each background and sample, and the spectra were obtained with a nominal resolution of $2 \mathrm{~cm}^{-1}$. In the HFIP samples solvent contribution was subtracted from the original spectra before the data analysis to allow a reliable comparison between spectra.

Data treatment and band decomposition of the original amide I have been described elsewhere ${ }^{59}$. In brief, the number and position of bands were obtained from the deconvolved (bandwidth $=18$ and $\mathrm{k}=2$ ) and the Fourier 
derivative (power $=3$ and breakpoint $=0.3$ ) spectra. The baseline was removed before starting the fitting procedure and initial heights set at $90 \%$ of those in the original spectrum for the bands in the wings and for the most intense component, and at $70 \%$ of the original intensity for the rest of bands. An iterative process followed, in two stages. (i) The band position of the component bands was fixed, allowing widths and heights to approach final values; (ii) band positions were left to change. For band shape a combination of Gaussian and Lorentzian functions was used. The restrictions in the iterative procedure were needed because initial width and height parameters can be far away from the final result due to the overlapping of bands, so that spurious results can be produced. In this way, information from band position, percentage of amide I band area and bandwidth were obtained for every component. Using this procedure the result was repetitive. Mathematical accuracy was assured by constructing an artificial curve with the parameters obtained and subjecting it to the same procedure again. The number of bands was fixed on the basis of the narrowing procedures. The molar absorption coefficient for the different bands was assumed to be similar and within a $+/-3 \%$ error.

To obtain the 2D-COS-IR maps, the Chol content was used to induce spectral fluctuations and to detect dynamic spectral variation in the secondary structure of CpreTM. Rendering of the two-dimensional synchronous and asynchronous spectra has been described previously ${ }^{69}$.

Attenuated total reflection IR spectroscopy (ATR-IR). ATR-IR spectra were measured in a Bruker Tensor 27 spectrometer equipped with a mercury-cadmium-telluride detector using a BioATRCell II microATR unit. $20 \mu \mathrm{l}$ of the lipid mixtures containing peptide were dried on the surface of the ATR Ge crystal by flowing dried air into the infrared spectrometer chamber during $5 \mathrm{~h}$. For spectra acquisition, the polarized mirror (Pike Technologies) was adjusted to $0^{\circ}$ and $90^{\circ}$, to generate incident light oriented parallel and perpendicular to the lipid normal, respectively. 150 IR spectra at $2 \mathrm{~cm}^{-1}$ resolution were collected under each condition and averaged. The dichroic ratio of the amide I bond absorption was computed for parallel $\left(0^{\circ}\right)$ versus perpendicular $\left(90^{\circ}\right)$ polarized incident light relative to the membrane normal and was employed to calculate the peptide orientation as discussed previously ${ }^{71,84,85}$.

Electron microscopy. As initial screen to determine the optimal concentration, samples were first imaged by negative stain electron microscopy. $8 \mu \mathrm{L}$ aliquots were adsorbed onto glow-discharged carbon coated copper grids, and negatively stained with $1 \%$ uranyl formate. Specimens were imaged with a JEM-1230 transmission electron microscope (JEOL Ltd. Tokyo) using an Orius SC1000 $(4008 \times 2672$ pixels $)$ cooled slow-scan CCD camera (Gatan Inc.) at the equivalent nominal magnification of $20000 x$, and defocus values between -2 and $-5 \mu \mathrm{m}$. Selected samples were then vitrified and imaged using a JEM-2200FSC transmission electron microscope (JEOL Ltd. Tokyo) equipped with a field emission gun (FEG) operated at $200 \mathrm{kV}$ and an in-column omega energy that helped us to record images with improved signal-to-noise ratio (SNR) by zero-loss filtering, using an energy selecting slit width of $30 \mathrm{eV}$ centered at the zero-loss peak of the energy spectra. Digital images were recorded under low dose conditions using a $4 \mathrm{~K} \mathrm{x} 4 \mathrm{~K}$ UltraScan $4000^{\mathrm{Tw}}$ charge-coupled device (CCD) camara (Gatan Inc.) at the equivalent nominal magnification of $50000 \mathrm{x}$, and defocus values between -1.5 and $-4 \mu \mathrm{m}$. $4 \mu \mathrm{L}$ aliquots were applied onto Quantifoil R 2/2 on 300 mesh cooper grids and C-flat R 1.2/1.3 on 400 mesh cooper grids plasma cleaned with air for $5 \mathrm{~s}$ using a PDC-002-CE plasma cleaner (Harrick Plasma). Grids were blotted and plunge frozen in liquid ethane with an automated Leica EM GP2 automatic plunge freezer (Leica Microsystems $\mathrm{GmbH}$, Wetzlar).

Received: 23 September 2020; Accepted: 13 December 2020

Published online: 14 January 2021

\section{References}

1. Melikyan, G. B. Membrane fusion mediated by human immunodeficiency virus envelope glycoprotein. Curr. Top. Membr. 68, 81-106. https://doi.org/10.1016/B978-0-12-385891-7.00004-0 (2011).

2. Blumenthal, R., Durell, S. \& Viard, M. HIV entry and envelope glycoprotein-mediated fusion. J. Biol. Chem. 287, 40841-40849. https://doi.org/10.1074/jbc.R112.406272 (2012).

3. Epand, R. M. Fusion peptides and the mechanism of viral fusion. Biochim. Biophys. Acta 1614, 116-121 (2003).

4. Apellaniz, B., Huarte, N., Largo, E. \& Nieva, J. L. The three lives of viral fusion peptides. Chem. Phys. Lipids 181, 40-55. https:// doi.org/10.1016/j.chemphyslip.2014.03.003 (2014).

5. Weissenhorn, W., Dessen, A., Harrison, S. C., Skehel, J. J. \& Wiley, D. C. Atomic structure of the ectodomain from HIV-1 gp41. Nature 387, 426-430 (1997).

6. Eckert, D. M. \& Kim, P. S. Mechanisms of viral membrane fusion and its inhibition. Annu. Rev. Biochem. 70, 777-810 (2001).

7. Buzon, V. et al. Crystal structure of HIV-1 gp41 including both fusion peptide and membrane proximal external regions. PLoS Pathog. 6, e1000880. https://doi.org/10.1371/journal.ppat.1000880 (2010).

8. Salzwedel, K., West, J. T. \& Hunter, E. A conserved tryptophan-rich motif in the membrane-proximal region of the human immunodeficiency virus type 1 gp41 ectodomain is important for Env-mediated fusion and virus infectivity. J. Virol. 73, 2469-2480 (1999).

9. Munoz-Barroso, I., Salzwedel, K., Hunter, E. \& Blumenthal, R. Role of the membrane-proximal domain in the initial stages of human immunodeficiency virus type 1 envelope glycoprotein-mediated membrane fusion. J. Virol. 73, 6089-6092 (1999).

10. Suarez, T., Gallaher, W. R., Agirre, A., Goni, F. M. \& Nieva, J. L. Membrane interface-interacting sequences within the ectodomain of the human immunodeficiency virus type 1 envelope glycoprotein: putative role during viral fusion. J. Virol. 74, 8038-8047 (2000).

11. Shang, L., Yue, L. \& Hunter, E. Role of the membrane-spanning domain of human immunodeficiency virus type 1 envelope glycoprotein in cell-cell fusion and virus infection. J. Virol. 82, 5417-5428. https://doi.org/10.1128/JVI.02666-07 (2008). 
12. Vishwanathan, S. A. \& Hunter, E. Importance of the membrane-perturbing properties of the membrane-proximal external region of human immunodeficiency virus type 1 gp41 to viral fusion. J. Virol. 82, 5118-5126 (2008).

13. Vishwanathan, S. A. et al. Hydrophobic substitutions in the first residue of the CRAC segment of the gp41 protein of HIV. Biochemistry 47, 124-130 (2008).

14. Zwick, M. B. The membrane-proximal external region of HIV-1 gp41: a vaccine target worth exploring. Aids 19, 1725-1737 (2005).

15. Montero, M., van Houten, N. E., Wang, X. \& Scott, J. K. The membrane-proximal external region of the human immunodeficiency virus type 1 envelope: dominant site of antibody neutralization and target for vaccine design. Microbiol. Mol. Biol. Rev. 72, 54-84 (2008).

16. Lorizate, M., Huarte, N., Saez-Cirion, A. \& Nieva, J. L. Interfacial pre-transmembrane domains in viral proteins promoting membrane fusion and fission. Biochim. Biophys. Acta 1778, 1624-1639 (2008).

17. Apellaniz, B. \& Nieva, J. L. The use of liposomes to shape epitope structure and modulate immunogenic responses of peptide vaccines against HIV MPER. Adv. Protein Chem. Struct. Biol. 99, 15-54. https://doi.org/10.1016/bs.apcsb.2015.03.002 (2015).

18. Contarino, M. et al. Chimeric Cyanovirin-MPER recombinantly engineered proteins cause cell-free virolysis of HIV-1. Antimicrob. Agents Chemother. 57, 4743-4750. https://doi.org/10.1128/AAC.00309-13 (2013).

19. Huarte, N. et al. Functional organization of the HIV lipid envelope. Sci. Rep. 6, 34190. https://doi.org/10.1038/srep34190 (2016).

20. Parajuli, B. et al. Lytic inactivation of human immunodeficiency virus by dual engagement of gp120 and gp 41 domains in the virus Env protein trimer. Biochemistry 55, 6100-6114. https://doi.org/10.1021/acs.biochem.6b00570 (2016).

21. Carravilla, P. et al. Effects of HIV-1 gp41-derived virucidal peptides on virus-like lipid membranes. Biophys. J. 113, 1301-1310. https://doi.org/10.1016/j.bpj.2017.06.061 (2017).

22. Cerutti, N., Loredo-Varela, J. L., Caillat, C. \& Weissenhorn, W. Antigp41 membrane proximal external region antibodies and the art of using the membrane for neutralization. Curr. Opin. HIV AIDS 12, 250-256. https://doi.org/10.1097/COH.000000000000036 4 (2017).

23. Montero, M. et al. Neutralizing epitopes in the membrane-proximal external region of HIV-1 gp41 are influenced by the transmembrane domain and the plasma membrane. J. Virol. 86, 2930-2941. https://doi.org/10.1128/JVI.06349-11 (2012).

24. Apellaniz, B. et al. The atomic structure of the HIV-1 gp41 transmembrane domain and its connection to the immunogenic membrane-proximal external region. J. Biol. Chem. 290, 12999-13015. https://doi.org/10.1074/jbc.M115.644351 (2015).

25. Lee, J. H., Ozorowski, G. \& Ward, A. B. Cryo-EM structure of a native, fully glycosylated, cleaved HIV-1 envelope trimer. Science 351, 1043-1048. https://doi.org/10.1126/science.aad2450 (2016).

26. Rujas, E. et al. Structural basis for broad neutralization of HIV-1 through the molecular recognition of 10E8 helical epitope at the membrane interface. Sci. Rep. 6, 38177. https://doi.org/10.1038/srep38177 (2016).

27. Chiliveri, S. C., Louis, J. M., Ghirlando, R., Baber, J. L. \& Bax, A. Tilted, uninterrupted, monomeric HIV-1 gp41 transmembrane helix from residual dipolar couplings. J. Am. Chem. Soc. 140, 34-37. https://doi.org/10.1021/jacs.7b10245 (2018).

28. Pinto, D. et al. Structural basis for broad HIV-1 neutralization by the MPER-specific human broadly neutralizing antibody LN01. Cell Host Microbe 26, 623-637. https://doi.org/10.1016/j.chom.2019.09.016 (2019).

29. Rantalainen, K. et al. HIV-1 envelope and MPER antibody structures in lipid assemblies. Cell Rep. 31, 107583. https://doi. org/10.1016/j.celrep.2020.107583 (2020).

30. Rujas, E. et al. Affinity for the interface underpins potency of antibodies operating in membrane environments. Cell Rep. 32, 108037. https://doi.org/10.1016/j.celrep.2020.108037 (2020).

31. Apellaniz, B., Ivankin, A., Nir, S., Gidalevitz, D. \& Nieva, J. L. Membrane-proximal external HIV-1 gp41 motif adapted for destabilizing the highly rigid viral envelope. Biophys. J. 101, 2426-2435. https://doi.org/10.1016/j.bpj.2011.10.005 (2011).

32. Apellaniz, B. \& Nieva, J. L. Fusion-competent state induced by a C-terminal HIV-1 fusion peptide in cholesterol-rich membranes. Biochim. Biophys. Acta 1014-1022, 2015. https://doi.org/10.1016/j.bbamem.2015.01.011 (1848).

33. Apellaniz, B. et al. Cholesterol-dependent membrane fusion induced by the gp41 membrane-proximal external region-transmembrane domain connection suggests a mechanism for broad HIV-1 neutralization. J. Virol. 88, 13367-13377. https://doi.org/10.1128/ JVI.02151-14 (2014).

34. Chernomordik, L. V. \& Kozlov, M. M. Protein-lipid interplay in fusion and fission of biological membranes. Annu. Rev. Biochem. 72, 175-207 (2003).

35. Akimov, S. A., Molotkovsky, R. J., Kuzmin, P. I., Galimzyanov, T. R. \& Batishchev, O. V. Continuum models of membrane fusion: evolution of the theory. Int. J. Mol. Sci. https://doi.org/10.3390/ijms21113875 (2020).

36. Jaroniec, C. P. et al. Structure and dynamics of micelle-associated human immunodeficiency virus gp 41 fusion domain. Biochemistry 44, 16167-16180. https://doi.org/10.1021/bi051672a (2005).

37. Li, Y. L. \& Tamm, L. K. Structure and plasticity of the human immunodeficiency virus gp41 fusion domain in lipid micelles and bilayers. Biophys. J. 93, 876-885 (2007).

38. Lai, A. L., Moorthy, A. E., Li, Y. \& Tamm, L. K. Fusion activity of HIV gp41 fusion domain is related to its secondary structure and depth of membrane insertion in a cholesterol-dependent fashion. J. Mol. Biol. 418, 3-15. https://doi.org/10.1016/j.jmb.2012.02.010 (2012).

39. Qiang, W., Sun, Y. \& Weliky, D. P. A strong correlation between fusogenicity and membrane insertion depth of the HIV fusion peptide. Proc. Natl. Acad. Sci. USA 106, 15314-15319. https://doi.org/10.1073/pnas.0907360106 (2009).

40. Saez-Cirion, A. \& Nieva, J. L. Conformational transitions of membrane-bound HIV-1 fusion peptide. Biochim. Biophys. Acta. 1564, $57-65$ (2002).

41. Martin, I. et al. Orientation and structure of the NH2-terminal HIV-1 gp41 peptide in fused and aggregated liposomes. Biochim. Biophys. Acta 1145, 124-133 (1993).

42. Martin, I., Schaal, H., Scheid, A. \& Ruysschaert, J. M. Lipid membrane fusion induced by the human immunodeficiency virus type 1 gp41 N-terminal extremity is determined by its orientation in the lipid bilayer. J. Virol. 70, 298-304 (1996).

43. Castano, S. \& Desbat, B. Structure and orientation study of fusion peptide FP23 of gp41 from HIV-1 alone or inserted into various lipid membrane models (mono-, bi- and multibi-layers) by FT-IR spectroscopies and Brewster angle microscopy. Biochim. Biophys. Acta 1715, 81-95. https://doi.org/10.1016/j.bbamem.2005.07.008 (2005).

44. Gabrys, C. M. et al. Solid-state nuclear magnetic resonance measurements of HIV fusion peptide CO to lipid P proximities support similar partially inserted membrane locations of the alpha helical and beta sheet peptide structures. J. Phys. Chem. A https://doi. org/10.1021/jp312845w (2013).

45. Gordon, L. M., Mobley, P. W., Pilpa, R., Sherman, M. A. \& Waring, A. J. Conformational mapping of the N-terminal peptide of HIV-1 gp41 in membrane environments using (13)C-enhanced Fourier transform infrared spectroscopy. Biochim. Biophys. Acta 1559, 96-120 (2002).

46. Martin, I. et al. Correlation between fusogenicity of synthetic modified peptides corresponding to the NH2-terminal extremity of simian immunodeficiency virus gp 32 and their mode of insertion into the lipid bilayer: an infrared spectroscopy study. J. Virol. 68, 1139-1148 (1994).

47. Mobley, P. W., Waring, A. J., Sherman, M. A. \& Gordon, L. M. Membrane interactions of the synthetic N-terminal peptide of HIV-1 gp41 and its structural analogs. Biochim. Biophys. Acta 1418, 1-18 (1999).

48. Rafalski, M., Lear, J. D. \& DeGrado, W. F. Phospholipid interactions of synthetic peptides representing the N-terminus of HIV gp41. Biochemistry 29, 7917-7922. https://doi.org/10.1021/bi00486a020 (1990). 
49. Qiang, W. \& Weliky, D. P. HIV fusion peptide and its cross-linked oligomers: efficient syntheses, significance of the trimer in fusion activity, correlation of beta strand conformation with membrane cholesterol, and proximity to lipid headgroups. Biochemistry $\mathbf{4 8}$, 289-301. https://doi.org/10.1021/bi8015668 (2009).

50. Nieva, J. L., Nir, S., Muga, A., Goni, F. M. \& Wilschut, J. Interaction of the HIV-1 fusion peptide with phospholipid vesicles: different structural requirements for fusion and leakage. Biochemistry 33, 3201-3209 (1994).

51. Pereira, F. B., Valpuesta, J. M., Basanez, G., Goni, F. M. \& Nieva, J. L. Interbilayer lipid mixing induced by the human immunodeficiency virus type-1 fusion peptide on large unilamellar vesicles: the nature of the nonlamellar intermediates. Chem. Phys. Lipids 103, 11-20 (1999).

52. Tristram-Nagle, S. et al. HIV fusion peptide penetrates, disorders, and softens T-cell membrane mimics. J. Mol. Biol. 402, 139-153. https://doi.org/10.1016/j.jmb.2010.07.026 (2010).

53. Ivankin, A., Kuzmenko, I. \& Gidalevitz, D. Cholesterol mediates membrane curvature during fusion events. Phys. Rev. Lett. 108, $238103(2012)$

54. Yao, H., Lee, M., Liao, S. Y. \& Hong, M. Solid-state nuclear magnetic resonance investigation of the structural topology and lipid interactions of a viral fusion protein chimera containing the fusion peptide and transmembrane domain. Biochemistry $\mathbf{5 5}$, 6787-6800. https://doi.org/10.1021/acs.biochem.6b00568 (2016).

55. Yao, H., Lee, M. W., Waring, A. J., Wong, G. C. \& Hong, M. Viral fusion protein transmembrane domain adopts beta-strand structure to facilitate membrane topological changes for virus-cell fusion. Proc Natl Acad Sci USA 112, 10926-10931. https://doi. org/10.1073/pnas.1501430112 (2015).

56. Lee, M. et al. Conformation and trimer association of the transmembrane domain of the parainfluenza virus fusion protein in lipid bilayers from solid-state NMR: insights into the sequence determinants of trimer structure and fusion activity. J. Mol. Biol. 430, 695-709. https://doi.org/10.1016/j.jmb.2018.01.002 (2018).

57. Liao, S. Y., Lee, M. \& Hong, M. Interplay between membrane curvature and protein conformational equilibrium investigated by solid-state NMR. J. Struct. Biol. 206, 20-28. https://doi.org/10.1016/j.jsb.2018.02.007 (2019).

58. Sanchez-Martinez, S. et al. Functional and structural characterization of 2B viroporin membranolytic domains. Biochemistry 47, 10731-10739. https://doi.org/10.1021/bi800997a (2008).

59. Arrondo, J. L. \& Goni, F. M. Structure and dynamics of membrane proteins as studied by infrared spectroscopy. Prog. Biophys. Mol. Biol. 72, 367-405 (1999).

60. Vu, D. M., Myers, J. K., Oas, T. G. \& Dyer, R. B. Probing the folding and unfolding dynamics of secondary and tertiary structures in a three-helix bundle protein. Biochemistry 43, 3582-3589. https://doi.org/10.1021/bi036203s (2004).

61. Walsh, S. T. et al. The hydration of amides in helices; a comprehensive picture from molecular dynamics, IR, and NMR. Protein Sci. 12, 520-531. https://doi.org/10.1110/ps.0223003 (2003).

62. Aloia, R. C., Tian, H. \& Jensen, F. C. Lipid composition and fluidity of the human immunodeficiency virus envelope and host cell plasma membranes. Proc. Natl. Acad. Sci. USA 90, 5181-5185 (1993).

63. Brügger, B. et al. The HIV lipidome: a raft with an unusual composition. Proc. Natl. Acad. Sci. USA 103, 2641-2646 (2006),

64. Campbell, S. M., Crowe, S. M. \& Mak, J. Virion-associated cholesterol is critical for the maintenance of HIV-1 structure and infectivity. AIDS 16, 2253-2261 (2002).

65. Guyader, M., Kiyokawa, E., Abrami, L., Turelli, P. \& Trono, D. Role for human immunodeficiency virus type 1 membrane cholesterol in viral internalization. J. Virol. 76, 10356-10364. https://doi.org/10.1128/jvi.76.20.10356-10364.2002 (2002).

66. Liao, Z., Graham, D. R. \& Hildreth, J. E. K. Lipid rafts and HIV pathogenesis: virion-associated cholesterol is required for fusion and infection of susceptible cells. AIDS Res. Hum. Retroviruses 19, 675-687. https://doi.org/10.1089/088922203322280900 (2003).

67. Fabian, H., Mantsch, H. H. \& Schultz, C. P. Two-dimensional IR correlation spectroscopy: sequential events in the unfolding process of the lambda cro-V55C repressor protein. Proc. Natl. Acad. Sci. USA 96, 13153-13158 (1999).

68. Noda, I. Two-dimensional correlation analysis useful for spectroscopy, chromatography, and other analytical measurements. Anal. Sci. 23, 139-146 (2007).

69. Iloro, I., Chehin, R., Goni, F. M., Pajares, M. A. \& Arrondo, J. L. Methionine adenosyltransferase alpha-helix structure unfolds at lower temperatures than beta-sheet: a 2D-IR study. Biophys. J. 86, 3951-3958 (2004).

70. Menestrina, G. Use of Fourier-transformed infrared spectroscopy for secondary structure determination of staphylococcal poreforming toxins. Methods Mol. Biol. 145, 115-132. https://doi.org/10.1385/1-59259-052-7:115 (2000).

71. Menikh, A., Saleh, M. T., Gariepy, J. \& Boggs, J. M. Orientation in lipid bilayers of a synthetic peptide representing the C-terminus of the A1 domain of shiga toxin. A polarized ATR-FTIR study. Biochemistry 36, 15865-15872. https://doi.org/10.1021/bi970944+ (1997).

72. Shai, Y. ATR-FTIR studies in pore forming and membrane induced fusion peptides. Biochim. Biophys. Acta 2306-2313, 2013. https ://doi.org/10.1016/j.bbamem.2012.11.027 (1828).

73. Serrano, S. et al. Structure-related roles for the conservation of the HIV-1 fusion peptide sequence revealed by nuclear magnetic resonance. Biochemistry 56, 5503-5511. https://doi.org/10.1021/acs.biochem.7b00745 (2017).

74. Yang, J., Gabrys, C. M. \& Weliky, D. P. Solid-state nuclear magnetic resonance evidence for an extended beta strand conformation of the membrane-bound HIV-1 fusion peptide. Biochemistry 40, 8126-8137 (2001).

75. Yang, J., Prorok, M., Castellino, F. J. \& Weliky, D. P. Oligomeric beta structure of the membrane-bound HIV-1 fusion peptide formed from soluble monomers. Biophys. J. 87, 1951-1963 (2004).

76. Schibli, D. J., Montelaro, R. C. \& Vogel, H. J. The membrane-proximal tryptophan-rich region of the HIV glycoprotein, gp41, forms a well-defined helix in dodecylphosphocholine micelles. Biochemistry 40, 9570-9578 (2001).

77. Dev, J. et al. Structural basis for membrane anchoring of HIV-1 envelope spike. Science 353, 172-175. https://doi.org/10.1126/ science.aaf7066 (2016).

78. Kwon, B., Lee, M., Waring, A. J. \& Hong, M. Oligomeric structure and three-dimensional fold of the HIV gp41 membrane-proximal external region and transmembrane domain in phospholipid bilayers. J. Am. Chem. Soc. 140, 8246-8259. https://doi.org/10.1021/ jacs.8b04010 (2018).

79. Sun, Z. Y. et al. HIV-1 broadly neutralizing antibody extracts its epitope from a kinked gp41 ectodomain region on the viral membrane. Immunity 28, 52-63 (2008).

80. Ofek, G. et al. Structure and mechanistic analysis of the anti-human immunodeficiency virus type 1 antibody $2 \mathrm{~F} 5$ in complex with its gp41 epitope. J. Virol. 78, 10724-10737 (2004).

81. Julien, J. P., Bryson, S., Nieva, J. L. \& Pai, E. F. Structural details of HIV-1 recognition by the broadly neutralizing monoclonal antibody 2F5: epitope conformation, antigen-recognition loop mobility, and anion-binding site. J. Mol. Biol. 384, 377-392 (2008).

82. Oakes, V. et al. Exposure of the HIV-1 broadly neutralizing antibody 10E8 MPER epitope on the membrane surface by gp41 transmembrane domain scaffolds. Biochim. Biophys. Acta Biomembr. 1259-1271, 2018. https://doi.org/10.1016/j.bbamem.2018.02.019 (1860).

83. Sreerama, N. \& Woody, R. W. Estimation of protein secondary structure from circular dichroism spectra: comparison of CONTIN, SELCON, and CDSSTR methods with an expanded reference set. Anal. Biochem. 287, 252-260 (2000).

84. Marsh, D. Dichroic ratios in polarized Fourier transform infrared for nonaxial symmetry of beta-sheet structures. Biophys. J. 72, 2710-2718. https://doi.org/10.1016/S0006-3495(97)78914-8 (1997).

85. Goormaghtigh, E., Raussens, V. \& Ruysschaert, J. M. Attenuated total reflection infrared spectroscopy of proteins and lipids in biological membranes. Biochim. Biophys. Acta. 1422, 105-185 (1999). 


\section{Acknowledgements}

This study was supported by the Spanish MCIU (Grants RTI2018-095624-B-C21; MCIU/AEI/FEDER, UE to JLN and BA; and PID2019-111096GA-I00; MCIU/AEI/FEDER, UE to AC) and Basque Government (Grant: IT1196-19). Technical assistance from MI Collado and M Carril with ${ }^{31} \mathrm{P}-\mathrm{NMR}$ measurements and data processing is greatly acknowledged.

\section{Author contributions}

I.D.-A., J.T. and J.L.N. conceived the experiments; I.D.-A., J.T., I.T., A.C., I.U.-B., J.L.R.A., .B.A. and J.L.N. designed them; I.D.-A. and J.T. prepared samples and determined structures by Infrared Spectroscopy; I.T., J.T. and I.U.-B. prepared and analyzed samples by Cryo-Electron Microscopy; A.C. and J.T. prepared and analyzed samples by Atomic Force Microscopy. J.L.N. wrote the paper with input from I.U.-B. and B.A., and all authors reviewed it.

\section{Competing interests}

The authors declare no competing interests.

\section{Additional information}

Supplementary Information The online version contains supplementary material availlable at https://doi. org/10.1038/s41598-020-80156-w.

Correspondence and requests for materials should be addressed to J.L.N.

Reprints and permissions information is available at www.nature.com/reprints.

Publisher's note Springer Nature remains neutral with regard to jurisdictional claims in published maps and institutional affiliations.

(c) (i) Open Access This article is licensed under a Creative Commons Attribution 4.0 International License, which permits use, sharing, adaptation, distribution and reproduction in any medium or format, as long as you give appropriate credit to the original author(s) and the source, provide a link to the Creative Commons licence, and indicate if changes were made. The images or other third party material in this article are included in the article's Creative Commons licence, unless indicated otherwise in a credit line to the material. If material is not included in the article's Creative Commons licence and your intended use is not permitted by statutory regulation or exceeds the permitted use, you will need to obtain permission directly from the copyright holder. To view a copy of this licence, visit http://creativecommons.org/licenses/by/4.0/.

(C) The Author(s) 2021 